\title{
Clustering and Path Planning for Wireless Sensor Networks based on Improved Ant Colony Algorithm
}

\author{
https://doi.org/10.3991/ijoe.v15i01.9784 \\ Jiajuan Fang \\ Zhengzhou Technical College, Zhengzhou, China \\ jiajuanfang7644@163.com
}

\begin{abstract}
To make up the deficiency of artificial intelligent ant colony algorithm in solving the clustering and path planning of wireless sensor network (WSN) a new random disturbance factor is proposed. A self-regulated random disturbance ant colony algorithm is obtained. An improved ant colony algorithm is proposed by combining the self-regulated random disturbance ant colony algorithm with chaos. After the algorithm improvement is completed, the improved artificial intelligent ant colony algorithm is applied to the cluster head fixed WSN node cluster and the path optimization process of each cluster head communication with the base station. The convergence speed, energy consumption and the survival time of the node cluster head are analyzed. The results show that the improved ant colony algorithm has good stability characteristics in the application and convergence of WSN. It can be seen that the improved ant colony algorithm is feasible in clustering and path planning of WSN.
\end{abstract}

Keywords-Ant colony algorithm, wireless sensor network, path planning

\section{Introduction}

As one of the current hot topics in the field of bionic intelligent optimization and artificial intelligence, the artificial intelligence ant colony algorithm has strong robustness and convergence, and it is easy to fuse with other algorithms. First of all, some artificial intelligence optimization algorithms are introduced, and the structure, principle, performance and characteristics of each algorithm are discussed. Then the artificial intelligent ant colony algorithm with excellent performance and suitable for wireless senor network (WSN) clustering is selected, and the defects of ant colony algorithm are improved. In the WSN cluster routing scene, the application of improved ant colony algorithm is divided into two cases: when the cluster head node is fixed, the improved ant colony intelligent optimization algorithm is used to divide clusters. When the cluster head collects the data, the data is sent to the base station in a multi-hop way. At this time, the path planning of the information sending is also implemented by the improved ant colony algorithm. When the sensor node of the system is elected as cluster head, the improved ant colony intelligent optimization 
Paper - Clustering and Path Planning for Wireless Sensor Networks based on Improved ...

algorithm is also used to iterate and complete the selection and cluster of cluster heads, and the simulation proves the advantage of the algorithm.

Through the research and analysis of the existing related literature, in order to solve the shortcomings of WSN clustering and path planning, the artificial ant colony algorithm is improved, and the improved ant colony algorithm is used to complete the clustering of WSN nodes and the path planning of the cluster starting data to the base station. Since the ant system has been put forward, many scholars have improved and applied it, and gradually overcome many shortcomings. Aiming at the problem of many iterations, low search efficiency and easy to fall into "premature" of artificial intelligence ant colony algorithm, an improved ant colony algorithm is proposed. Firstly, an adaptive disturbance factor is proposed on the basis of the special ant colony strategy and the disturbance ant colony algorithm. As a result, it speeds up the convergence speed of the algorithm, and also improves the premature stagnation of the algorithm and obtains self-regulated random disturbance ant colony algorithm. In the meanwhile, in order to further improve the stagnation of ant colony algorithm, an improved ant colony algorithm is obtained combining self-regulating random disturbance ant colony algorithm with chaos strategy, and the best selection solution for the chaotic parameter in this algorithm is proposed. When the ant colony is improved, the improved ant colony algorithm is applied to the cluster routing model of WSN. Here, the cluster head of WSN is fixed after the cluster head is distributed, and the cluster head is not replaced with rotation. The sensor nodes join the cluster head by the improved ant colony algorithm, and the routing planning of the cluster head to the base station is also completed by the improved ant colony algorithm.

\section{$2 \quad$ Literature Review}

The basic idea of WSN originated in 1970s. Visser and Vullers (2013) mentioned in the research that the United State Vision Program Research Bureau has invested tens of millions of dollars to help universities and students to explore WSN technology. In 2003, NSF made detailed plans for WSN, and invested greatly in research projects, and a research center related to WSN was also set up at California University [1]. Xie and Wang (2014) believed that nodes in WSNs were often distributed in remote areas, field, battlefield, or areas people difficult to reach. Usually, battery provided the necessary energy for the node, its energy would be depleted, and people could not replace the battery or charge of nodes to extend the life of the node [2]. Chu et al. (2014) thought that the WSN clustering routing scheme first needed to consider improving the utilization of energy and reducing energy consumption. According to the different application objectives, WSN's clustering routing protocol mechanism would also have different rendering [3]. According to the characteristics of the cluster routing mechanism adopted by the application objects, different methods are used to distinguish the wireless routing protocols.

As a low-energy and adaptive hierarchical clustering routing protocol, the LEACH protocol dynamically selects common sensor nodes as cluster heads, thus balancing the load of network energy. However, there are some randomness and uncertainty in 
Paper - Clustering and Path Planning for Wireless Sensor Networks based on Improved ...

the generation of cluster heads and the number of cluster heads in the protocol. The nodes in the network are randomly elected as cluster heads, and the energy consumption of the nodes is not considered. The cluster heads which are far away from the base station will die early because of the premature depletion of energy. In view of the above shortcomings, Ma et al. (2013) proposed LEACH-C, and LEACH$\mathrm{C}$ made some improvements to LEACH. The cluster structure of the LEACH-C protocol was generated by the base station through simulated annealing algorithm [4]. Since the cluster heads selected by the LEACH-C protocol send information in the form of one or more hops to the base station, this will result in the problem that the cluster heads near the base station will receive and forward the data too much, which will consume more energy and shorten the lifetime. The hybrid, energy-efficient distributed clustering protocol determines the radius of each cluster. The basis for the protocol to select the cluster head set is the residual energy of nodes and followed by the density of nodes. For the main basis, the node with more remaining energy is more likely to be elected as the cluster head of this round; for the secondary basis, that is the degree of proximity between the nodes or the density of the nodes in the network. For cluster heads in the same cluster coverage area, they have the same rights to run as the final cluster heads through the average reachable parameter (AMRP). For cluster members that exist in a number of cluster coverage areas, different strategies are applied. They refer to sub parameter AMRP to add the final cluster. The LEACH protocol has another disadvantage that the protocol neither calculate nor refer to the residual energy of each sensor node when the cluster head set is elected. If the nodes with little remaining energy are selected as cluster heads, the energy consumption will be greatly increased, the energy will be exhausted too early, and the operation of the whole network will be greatly affected. In view of this defect, Sun and Jian-Zhong (2013) took the remaining energy of the node into consideration when selecting the cluster head, and solved the defect of the LEACH protocol [5].

The routing protocol based on swarm intelligence focuses on the behavior of the whole group, and emphasizes the overall situation compared with the hierarchical protocol. The artificial intelligence ant colony algorithm is a parallel self-organizing algorithm, which has strong robustness and has a positive feedback mechanism. These features make it suitable for application in the WSN environment. Shelke et al. (2016) combined ant colony algorithm with LEACH protocol, and proposed a unicast routing algorithm which can be used in WSNs. This algorithm was applied to deal with the problems in large-scale WSN environment [6]. Kefayat et al. (2015) applied ant colony algorithm to the cluster phase of WSN, and put forward a double cluster head algorithm based on ant colony, which made the different cluster heads perform different work, so as to achieve the balance of energy using [7]. Fard and Monfaredi (2015) used the artificial intelligence ant colony algorithm to the stable communication stage of data information and proposed a LEACH algorithm based on ant colony algorithm. They counted the energy consumption of the cluster heads and calculate the distance of the adjacent cluster heads. After processing the above information, they are counted into the pheromone of the ant colony algorithm to form a multi-hop optimal path [8]. Related scholars proposed an ant colony based 
Paper - Clustering and Path Planning for Wireless Sensor Networks based on Improved ...

clustering routing algorithm for WSNs. This algorithm considers the distance threshold when clustering the network, calculates the residual energy of the nodes, and selects the nodes with great remaining energy to become the cluster head. As a result, the distribution of cluster heads is more reasonable, and the ant colony algorithm is applied to the data communication stage to provide the most reasonable transmission scheme for all cluster heads to reach the base station. A LEACH protocol based on ant colony algorithm is proposed by the relevant scholars, which first considers the number of nodes to determine the area of the cluster. After that, the energy of each node is counted and elected as cluster head if the residual energy is higher than the set value. The search angle is determined at the communication stage. The ant colony algorithm iterates to get the most reasonable path from cluster head to sink node. A WSN routing algorithm based on ant colony optimization is proposed by some scholars. The algorithm stipulates that the nodes with more residual energy have the opportunity to be elected as cluster heads. The residual energy and distance of the nodes adjacent to these nodes are counted, and the sink node calculates and plans the clusters and the next hop information of the nodes.

To sum up, the artificial intelligence algorithm can effectively complete the clustering and path planning of WSNs. The artificial ant colony algorithm imitates the foraging process of the ants in nature, and it has a forward feedback, distributed parallel computer system, and strong robustness. Through the research and analysis of the existing related literature, in order to solve the shortcomings of WSN clustering and path planning, an improved scheme is proposed, so as to further improve the energy efficiency.

\section{Method}

\subsection{Special ant colony algorithm}

In the genetic algorithm, after the sequence crossover and mutation of the algorithm, the optimal sequence of the previous generation will change, and it is difficult to keep it to the next generation. Then, the information of the most adapted sequence of the previous generation will be lost. In view of this, a special ant colony algorithm is proposed to solve this problem. The special ant colony algorithm enhances the positive feedback of the best sequence obtained in each iteration of the algorithm, improves the pheromone updating method of the self-regulating random disturbance ant colony algorithm, and adds the additional pheromone to the original pheromone updating formula. Based on the above thought, this section uses the literature for special ant colony optimization algorithm to improve the basic ant colony algorithm. After completing one iteration, the related parameters of current optimal sequence are recorded, and the influence of the current optimal sequence is strengthened during the update of pheromone. Pheromone updating is shown in Formula (1):

$$
\tau_{j}(t)=(1-\rho) \cdot \tau_{j}(t-1)+\Delta \tau_{j}+\Delta \tau_{j}^{*} .
$$


Paper - Clustering and Path Planning for Wireless Sensor Networks based on Improved ...

$$
\Delta \tau_{j}^{*}=\left\{\begin{array}{ll}
\frac{R}{L^{*}} & \text { If cluster head } \mathrm{J} \text { is part of the local optimal solution identified } \\
0 & \text { Otherwise }
\end{array} .\right.
$$

In the above formulas, $\Delta \tau^{*}{ }_{\mathrm{j}}$ suggests the pheromone additionally added by the ant colony algorithm on the cluster head $\mathrm{j}, \mathrm{R}>0$ indicates the parameter, and $\mathrm{L}^{*}$ represents the total length of the optimal sequence covered by WSN in this iteration.

\subsection{Self-regulating random disturbance ant colony algorithm}

The artificial intelligence ant colony algorithm has a strong global performance and a certain feedback mechanism. However, its complex transfer probability formula increases the difficulty of iterative computation, and it is possible to fall into the local optimal in the iterative process of the algorithm. In this respect, first of all, the random perturbation transfer strategy is used to simplify the transfer probability formula referring to the idea of predecessors. Based on a random disturbance transfer strategy, a self-regulating random disturbance factor is proposed to improve the shortcoming of premature stagnation of the algorithm, so that the algorithm is more global.

$\eta_{\mathrm{ij}}(\mathrm{t})$ in the transfer probability formula represents the heuristic information of the ant colony, which reflects the heuristic degree of initialization of the ant colony algorithm. In the cluster routing problem, each sensor node is independent of each other, so the heuristic information $\eta_{\mathrm{ij}}(\mathrm{t})$ can be directly abandoned from the transfer probability. The change of the pheromone $\eta_{\mathrm{ij}}(\mathrm{t})$ on each path is sufficient to express the change of the transfer probability. Therefore, after removing the heuristic information, the transfer probability formula of ant colony algorithm can be improved and simplified.

$$
P_{i j}^{k}(t)=\left\{\begin{array}{ll}
\frac{\tau_{i j}(t)}{\sum_{s \in J_{k}(i)} \tau_{i s}(t)} & \text { if } j \in J_{k}(i) \\
0 & \text { else } .
\end{array} .\right.
$$

There are two parameters in the heuristic information, which are heuristic factor $\alpha$ and expectation heuristic factor $\beta$, which can be obtained by observing Formula (3). When ignoring the heuristic information $\eta_{\mathrm{ij}}(\mathrm{t})$, the two parameters are also ignored. As a result, the Formula (3) is only influenced by $\tau_{\mathrm{ij}}(\mathrm{t})$. The transfer probability in the Formula (3) is compared with the random number of $(0,1)$. If the transfer probability is larger, then the ant passes through the $\mathrm{j}$ node; otherwise the ant does not pass through the node. The scheme not only simplifies the formula, but also reduces the complexity of the calculation, and the difficulty of parallel computing is very low, even in the face of large scale data due to the independence of node acquisition data.

After simplifying the transfer probability function of ant colony algorithm, the computational complexity is reduced and it is suitable for parallel computing. However, the time spent in the search of the optimal solution is still very long, and the premature stagnation still appears in the iterative process of the algorithm. Aiming at 
Paper — Clustering and Path Planning for Wireless Sensor Networks based on Improved ...

the shortcomings of the ant colony algorithm, many scholars at home and abroad have gained a lot of achievements and progress through a great deal of work and research, such as dynamic ant colony and system with elite strategy. The relevant scholars proposed ant colony optimization with random perturbation behavior. Based on the algorithm proposed in this literature, a new disturbance factor is proposed here for the above shortcomings, and the self-regulating random disturbance ant colony algorithm is obtained. The simulation results show that the algorithm has good random disturbance characteristics and convergence stability characteristics.

The transfer probability function of the basic ant colony algorithm is studied. And this function is used as the characteristic of the ant search for the optimal path to get a transfer strategy with random disturbance:

$$
C_{i j(k)}= \begin{cases}\left(\tau_{i j(k)} \eta_{i j(k)}\right)^{\gamma} & j \notin T a b u_{(k)} \\ 0 & \text { Otherwise } .\end{cases}
$$

In the above formula, the disturbance factor $\gamma>0 . \mathrm{C}_{\mathrm{ij}(\mathrm{k})}$ in Formula (4) represents the transfer coefficient on the cluster head path $(i, j)$, not the "transfer probability", and each path has its own transfer coefficient. When ants choose the optimal path, the maximum coefficient is usually chosen, thus it can be seen that the coefficient can be considered as a definite value. After the analysis and discussion of the algorithm, it is found that: after the improvement of the ant colony algorithm, the coefficient $C_{\mathrm{ij}(\mathrm{k})}$ is obtained. If the coefficient $\gamma$ is fixed, the improved ant colony algorithm shows the same performance as the pre-improved algorithm, and the stagnation phenomenon will appear in the iterative process. In order to solve this problem, it is expected that the coefficient $\mathrm{C}_{\mathrm{ij}(\mathrm{k})}$ changes with the iterative process to adapt to the algorithm, and the following two points are considered to design the disturbance factor of the transfer coefficient:

First, in the case of considering that the number of ants is very large, the ant individual is more inclined to choose a path with greater transfer coefficient when choosing the path. But there is a lot of disorder path for it to choose, so it is more difficult to choose a better path in a short time. Therefore, larger perturbation factors are used, which improves the concentration of pheromones on relatively excellent paths, and accelerates the convergence speed in the algorithm iteration process.

Second, if the disturbance factor remains the same, the pheromone on a path in the iterative process will accumulate continuously, and the pheromone concentration on this path will be far higher than that of the other path. This path may not be global optimal, which may lead to a stagnation in the subsequent iterative search process. Therefore, a self-regulating disturbance factor can be used to make it smaller in the search process. As a result, the probability of selecting the optimal path will increase and the convergence speed will become more gentle.

Based on the above two points, a self-regulating disturbance factor is proposed, as shown in Formula (5), which is effective.

$$
\gamma=\frac{a \pi}{2 \arctan k} \quad k=1,2,3 \ldots \ldots N C_{\max } .
$$


Paper — Clustering and Path Planning for Wireless Sensor Networks based on Improved ...

In the formula: $\mathrm{NC}_{\max }$ represents the maximum number of iteration; a is the scale parameter of the self-regulating disturbance factor. From Formula (5), it can be obtained that, as the algorithm iterates continuously, the perturbation factor $\gamma$ will gradually approach to a.

The self-regulating disturbance factor is used to reduce the local optimal solution in the iterative calculation process of the ant colony algorithm, and the improved scheme is proposed. However, in the iterative process of the algorithm, the optimal path may be omitted because of the uncertainty of the path selection. In order to avoid this phenomenon, and in the algorithm process, the random selection probability can be adjusted with the change of the number of iterations, the modification scheme of the transfer probability coefficient of the related literature is put forward:

$$
C_{i j(k)}=\left\{\begin{array}{ll}
\left(\tau_{i j k} \eta_{i j(k)}\right)^{\gamma} & \tau_{i j(k)}=\max \left(\tau_{i s(k)}\right), s \notin T a b u_{(k)} \\
\left(\tau_{i j k}\right)^{\alpha} \eta_{i j(k)} & P_{m} \geq U, s \notin T a b u_{(k)} \\
\left(\tau_{i j k k} \eta_{i j(k)}\right)^{\gamma} & P_{m} \prec U, s \notin T a b u_{(k)} \\
0 & \text { Otherwise }
\end{array} .\right.
$$

In Formula (6): $\mathrm{P}_{\mathrm{m}} \in(0,1)$ is a random mutation rate; $\mathrm{U}$ is a uniformly distributed random number in $(0,1)$. Formula (6) shows that for the path with the highest pheromone and when the random variation rate is less than the random number, the algorithm uses the general transfer coefficient formula. When the random mutation rate is larger than the random number produced, the algorithm will use the random mutation transfer coefficient formula. Bu using this scheme, not only the path of the maximum transfer coefficient is included, but also the randomness of the algorithm is strengthened, and the global searching ability of the algorithm is strengthened.

After the improvement of the transfer probability of the basic ant colony algorithm, the improved algorithm is applied to the cluster problem model. Besides the transfer probability, the other parts of the algorithm remain unchanged. On the basis of the simplification of the clustering algorithm of the ant colony optimization, the heuristic information $\eta_{\mathrm{ij}}(\mathrm{t})$ and the parameters $\alpha$ and $\beta$ are ignored, and the transfer coefficient of Formula (6) can be expressed as:

$$
C_{i j(k)}= \begin{cases}\left(\tau_{i j k(k)}\right)^{\gamma} & P_{m} \geq U \\ \tau_{i j(k)} & P_{m} \prec U .\end{cases}
$$

At this point, the improvement of the self-regulating random disturbance ant colony algorithm has been completed. Next, this algorithm will be combined with chaos to further improve the algorithm.

\subsection{Application of improved ant colony algorithm to clustering WSNs}

Clustering algorithm is different from existing protocols such as LEACH. When a number of sensors are distributed randomly, the cluster heads are determined beforehand. The objective is to rationally allocate the non-cluster head sensor nodes to different cluster heads to form a cluster, so as to achieve the lowest energy consumption or to balance the energy consumption of the network nodes. 
Paper — Clustering and Path Planning for Wireless Sensor Networks based on Improved ...

When the sensor and cluster head nodes are randomly distributed, the cluster head nodes broadcast their position to the network. The selection stage of the cluster heads is calculated by the improved artificial intelligent ant colony algorithm, and the cluster is completed. After forming a complete cluster, the cluster head counts the number of cluster members and sets up the scheduling table. Then, it enters the information transmission stage. In the cluster, the cluster head receives the information sent by the cluster members and compresses the received data. Outside the cluster, each cluster head transfers data to the base station by multi-hop, and the transmission path of cluster heads is also obtained by improved artificial intelligence ant colony algorithm. Therefore, the problem of clustering is discussed first here.

Assuming that WSN has $\mathrm{N}$ sensor nodes and $\mathrm{M}$ cluster heads, the positions of nodes and cluster heads are fixed after distribution and the WSN clustering iteration process is as follows:

Step 1: Calculate the distance between each sensor node and cluster head. If the distance between nodes and cluster heads is larger than the communication distance of sensor nodes, then this node cannot join the corresponding cluster heads.

Step 2: Each iteration produces $M$ candidate solutions, the dimension of the candidate solution is $\mathrm{N}$, and each dimension of the solution corresponds to a member node. Each node selects the cluster head in its own communication range through chaos initialization and joins it into its member node. Then, the fitness function of each cluster is calculated.

Step 3: Each cluster head is a member node that sets each city on the ant colony. The improved artificial intelligent ant colony algorithm is implemented to update the cluster head member nodes to achieve the optimal.

Step 4: The number of iterations of the algorithm adds one and judge whether it has reached the maximum number of iteration (convergence). If not, then go to step 2; if the maximum number of iterations is reached, then the algorithm ends, and the optimal clustering scheme is recorded by the improved ant colony algorithm in the solution process.

\section{$4 \quad$ Results}

Experiment 1: Hop number and path coverage distance between cluster heads and base stations in the cluster routing scheme of each algorithm.

Starting from the hop number of the cluster head and the total road coverage, compared with the basic ant colony algorithm, the improved algorithm has no greater improvement. But it is worth noting that, when the number of cluster heads becomes larger, the improved ant colony algorithm shows the advantages slowly. As shown in Figure 1 and Figure 2, the routing coverage distance is greater at 60 and 70 cluster heads than that of basic ant colony algorithm routing scheme when the cluster head node changes from less to more. When it reaches 80 or 90 , the path of the routing scheme coverage of the improved ant colony algorithm is better than that of the basic ant colony algorithm routing scheme. 
Paper - Clustering and Path Planning for Wireless Sensor Networks based on Improved ...

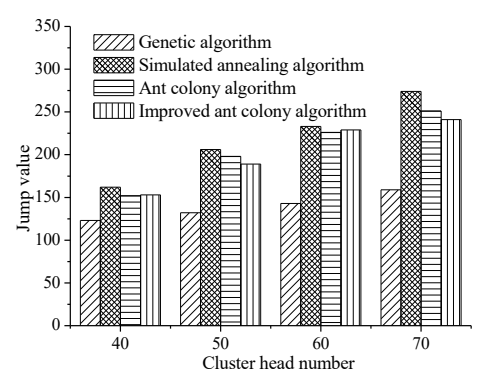

Fig. 1. Comparison of cluster head hop number of artificial intelligence algorithm

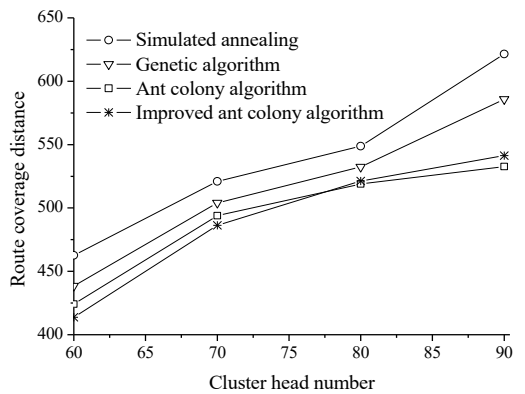

Fig. 2. The path of artificial intelligence algorithm cluster head routing coverage

Experiment 2: The convergence of the improved ant colony algorithm is compared with that of the basic ant colony algorithm.

Figure 3 shows the comparison of the convergence of the fitness function between the improved ant colony algorithm and the basic ant colony algorithm. It can be seen that the improved ant colony algorithm converges after 30 iterations and reaches the fitness value of 0.225 ; the basic ant colony algorithm converges after 50 iterations, and the value of the fitness function is 0.2 . It is concluded that the ant colony algorithm improved in clustering has some advantages compared with the basic ant colony algorithm.

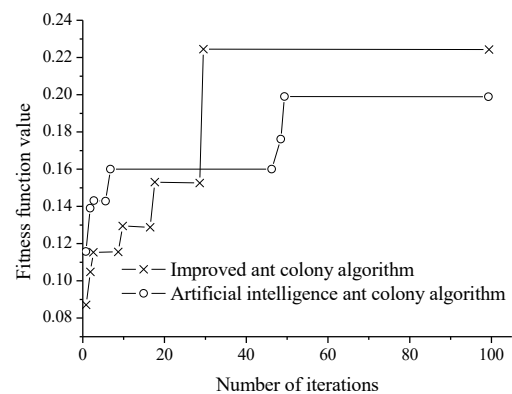

Fig. 3. The comparison of the convergence of the fitness function between the improved ant colony algorithm and the basic ant colony algorithm 
Paper - Clustering and Path Planning for Wireless Sensor Networks based on Improved ...

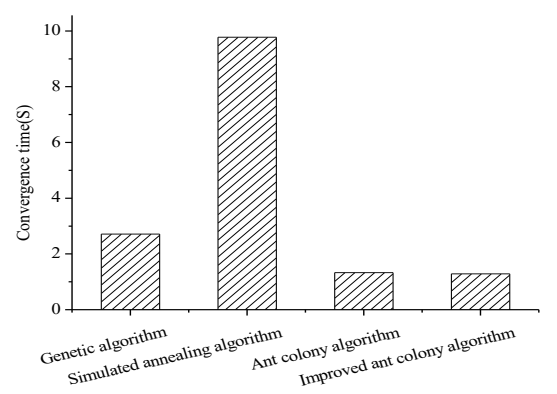

Fig. 4. Time for each intelligent algorithm to complete the WSN clustering

Figure 4 shows the time for each intelligent algorithm to complete the WSN clustering. The improved ant colony algorithm takes only 1.28 seconds, obviously better than the genetic algorithm and the simulated annealing algorithm, and has a certain improvement compared with the ant colony algorithm.

As for the fitness function of the routing algorithm, it is composed of the total path covered by the path and the number of cluster head jumps, so the smaller the fitness is, the more it is in accordance with the requirements. As shown in Figure 5, the improved ant colony algorithm converges after 11 iterations, and the basic ant colony algorithm converges after 17 iterations and the fitness function value obtained are not as good as the improved ant colony algorithm.

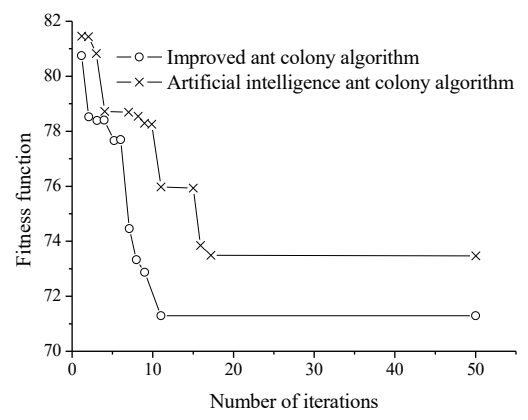

Fig. 5. Comparison of fitness function convergence between improved ant colony algorithm and basic ant colony algorithm in route planning algorithm

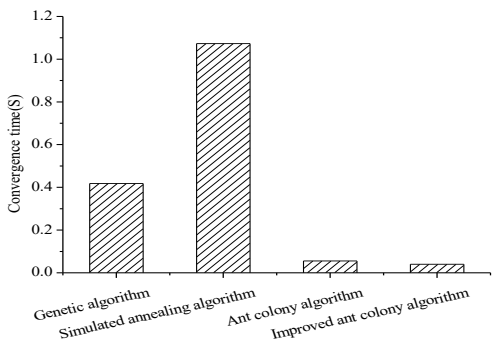

Fig. 6. Time for each algorithm to complete routing planning 
Paper — Clustering and Path Planning for Wireless Sensor Networks based on Improved ...

Figure 6 gives the convergence time for an improved ant colony algorithm to complete the WSN routing planning. Its convergence rate is far superior to that of genetic algorithm and simulated annealing algorithm, and is slightly better than that of ant colony algorithm. It can be seen that the improved ant colony algorithm is superior in the convergence time.

Experiment 3: The energy consumption of the improved ant colony algorithm is compared with that of other algorithms.

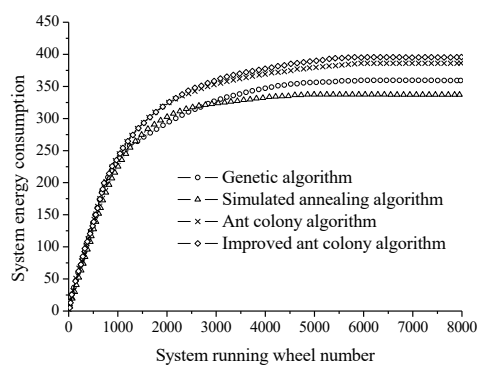

Fig. 7. The change of the system energy consumption with the operation of the system

The residual energy and the survival time of the sensor are the important indexes for evaluating the clustering scheme. Figure 7 clearly shows that, with the change of the remaining energy of the system's operating system, the improved ant colony algorithm consumes $396.75 \mathrm{~J}$ energy before the system is paralyzed, which is better than $383.21 \mathrm{~J}$ of the ant colony algorithm, 360.53J of the genetic algorithm, and $343.95 \mathrm{~J}$ of the simulated annealing algorithm. It can be seen that the improved ant colony algorithm is more reasonable, so that the system can make full use of the system energy and run longer.

Experiment 4: The clustering scheme of improved ant colony algorithm is compared with the scheme of other algorithms in terms of the system node death graph.

In Figure 8, the network obtained by the cluster scheme of the basic ant colony algorithm shows the death node when the system runs 3550 times. The improved ant colony algorithm shows the death node at 3700 , and the number of node deaths is less than that of the basic ant colony algorithm. Thus, the clustering scheme obtained by the improved ant colony algorithm can extend the life of the system nodes and improve the system performance. 
Paper - Clustering and Path Planning for Wireless Sensor Networks based on Improved ...

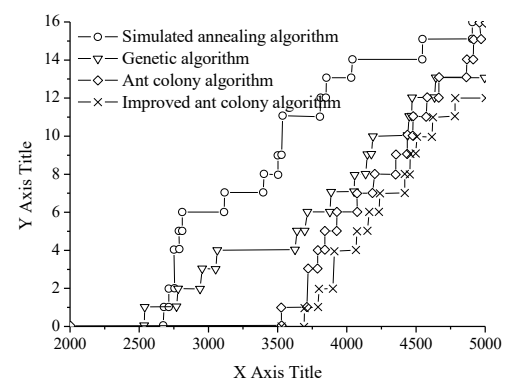

Fig. 8. The change of the number of dead nodes with the times of the system operation

Experiment 5: The clustering scheme obtained by improved ant colony algorithm is compared with the scheme obtained by other algorithms in terms of cluster head death graph.

As for the implementation of routing algorithms, the death trend of cluster heads is an important performance index. As shown in Figure 9, the routing scheme obtained by the basic ant colony algorithm begins to have death cluster head after the system runs 1150 times. The routing scheme obtained by improved ant colony algorithm begins to have death cluster head only when the system runs 2050 times. Moreover, the survival time of cluster heads of clustering routing scheme obtained by improved ant colony algorithm is much larger than that of other schemes.

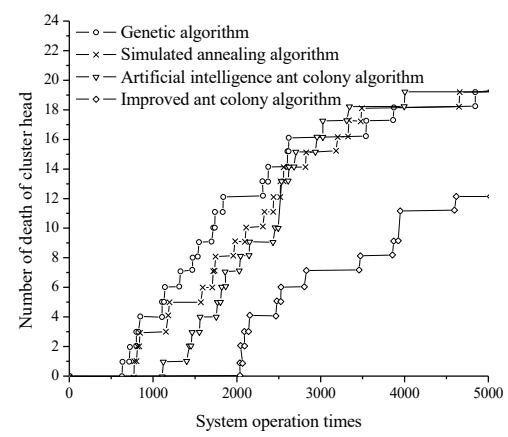

Fig. 9. The change of the number of death of cluster head with the times of the system operation

The simulation results show that, compared with the basic ant colony algorithm, the improved ant colony algorithm not only simplifies the algorithm, but also improves the performance of the clustering of WSNs and the path optimization of the cluster heads. 
Paper - Clustering and Path Planning for Wireless Sensor Networks based on Improved ...

\section{Conclusion}

On the basis of a series of improved ant colony algorithm for artificial intelligence proposed, a new random disturbance factor is proposed, and the self-regulating random disturbance ant colony algorithm is obtained. The ant colony algorithm of random disturbance makes the algorithm more balanced. The special ant colony algorithm is to make the original algorithm strengthen the forward feedback of the optimal sequence obtained by the current iteration. At the same time, aiming at the stagnation in the iterative process of the algorithm, an improved ant colony algorithm is proposed by combining the self-regulating random disturbance ant colony algorithm and chaos. In addition, the optimal parameter selection scheme of the chaotic motion in this algorithm is proposed by simulation. After the improvement of the algorithm, the improved artificial intelligent ant colony algorithm is applied to the WSN node clustering with fixed cluster head and the path optimization of the communication between the cluster heads and the base station. The convergence speed, the energy consumption and the survival time of the node cluster head are analyzed, and the advantages and convergence stability of improved ant colony algorithm in WSN are proved.

\section{References}

[1] Visser, H.J., Vullers, R.J.M. (2013). RF Energy Harvesting and Transport for Wireless Sensor Network Applications: Principles and Requirements. Proceedings of the IEEE, 101(6): 1410-1423. https://doi.org/10.1109/JPROC.2013.2250891

[2] Xie, S, Wang, Y. (2014). Construction of Tree Network with Limited Delivery Latency in Homogeneous Wireless Sensor Networks. Wireless Personal Communications, 78(1): 231246. https://doi.org/10.1007/s11277-014-1748-5

[3] Chu, S.I., Lien, C.Y., Lin, W.C. (2014). A Survey of Localization in Wireless Sensor Network. International Journal of Distributed Sensor Networks, 2012(1): 385-391.

[4] Ma, R., Liu, Y., Wang, C. (2013). An Energy Efficient and Reliable Routing Protocol Using Clustering Hierarchical Structure for Wireless Sensor Networks. International Journal of Digital Content Technology \& Its Applications, 7(3): 761-769. https://doi.org/10.4156/jdcta.vol7.issue3.91

[5] Sun, Y, Jian-Zhong, H.E. (2013). Adaptive load-balancing clustering hierarchical routing protocol for WSN. Computer Engineering \& Design, 34(2): 423-427.

[6] Shelke, M., Tefera, G., Mahalle, P. (2016). Fuzzy-based fault-tolerant low-energy adaptive clustering hierarchy routing protocol for wireless sensor network. International Journal of Wireless \& Mobile Computing, 11(2): 117-123. https://doi.org/10.1504/IJWMC.2016.080183

[7] Kefayat, M., Ara, A.L., Niaki, S.A.N. (2015). A hybrid of ant colony optimization and artificial bee colony algorithm for probabilistic optimal placement and sizing of distributed energy resources. Energy Conversion \& Management, 92(3): 149161. https://doi.org/10.1016/j.enconman.2014.12.037

[8]Fard, E.S., Monfaredi, K. (2015). Optimization of the running speed of ant colony algorithm with address-based hardware method. Genetika, 16(5): 783-6. 
Paper - Clustering and Path Planning for Wireless Sensor Networks based on Improved ...

\section{$7 \quad$ Author}

Jiajuan Fang is a Researcher of Zhengzhou Technical College, Zhengzhou, Henan 450121, China. His research interests include ant colony algorithm.

Article submitted 28 October 2018. Resubmitted 25 November 2018. Final acceptance 12 December 2018. Final version published as submitted by the authors. 